\title{
Prediction of Retail Beef Yield Using Parameters Based on Korean Beef Carcass Grading Standards
}

\author{
Yun Ho Choy*, Seong Bok Choi, Gi Jun Jeon, Hyeong Cheol Kim, Hak Jae Chung, \\ Jong Moon Lee, Beom Young Park, and Sun Ho Lee ${ }^{1}$ \\ National Institute of Animal Science, RDA, Suwon 441-706, Korea \\ ${ }^{1}$ Korea Institute for Animal Products Quality Evaluation, Gunpo 435-010, Korea
}

\begin{abstract}
Two sets of data on carcass traits and beef cut parameters were used to investigate the relationships between carcass and beef cut measurements, which can be used to make predictions of retail cut percentages. One set had a total of 1,141 measurements of Hanwoo cattle of three different sex origins, which were slaughtered in an abattoir located at the National Institute of Animal Science, RDA, Korea from 1996 to 2008. To develop prediction models for retail cut percentage with higher accuracies than the current model, another set consisting of a total of 13,389 records of carcass and beef cut traits were collected from 30 abattoirs and butcheries in Korea from 2008 to 2009. Bulls yielded heavier and leaner carcasses than steers. High correlation coefficients were estimated between amount of body fat and percent retail cut (-0.82) as well as between back fat thickness (BF) and percent retail cut (-0.62). The amount of retail cut, however, was highly correlated with body weight before slaughter (BW, 0.95 ) or with cold carcass weight (CWT, 0.94). Relationships between percent retail cut and measurable beef yield traits, BF, loin eye area (LEA) or CWT varied by sex class, which must be considered for development of a prediction model with high accuracy. Models of data for all breeds and sexes fit the effects of breed, sex, and interaction of abattoir by butchers, whereas models of data for each breed and sex fit the effect of interaction of abattoir by butcher only. Due to possible future changes in back fat control, we performed a log transformation of BF. Our new models fit better than the currently used model.
\end{abstract}

Key words: carcass, Hanwoo, retail cut percentage, yield grade, breed, sex

\section{Introduction}

Cattle breeds in Korea, which are used for beef production, consist of Hanwoo (Korean Cattle), Holstein steers, some European breeds and some crossbreds of two major breeds. Holstein cattle are widely grown as dairy cattle but their male progenies (steers) are used for meat consumption. Therefore, breeding objectives of Holstein breed are mainly milk production, yield and quality. On the other hand, Hanwoo cattle are mostly bred for beef production. National breeding objectives also focus on their beef yield and quality. As consumers' growing concern on more tasteful beef products, Hanwoo breeding goals now focus more on beef quality than on yield. This shift in genetic change affects other economically important characteristics such as back fat or retail cut yield,

*Corresponding author: Yun Ho Choy, Animal Breeding \& Genetics Division, National Institute of Animal Science, RDA, Choenan 232-952, Korea. Tel: 82-41-580-3354; Fax: 82-41582-1248; E-mail: ychoy000@korea.kr which are directly related to profitability from respect of beef salers. Retail beef cut percentage is a major economical interest especially to beef salers. Prediction of retail beef yield or the ratio with respect to carcass weight costs high and cannot be made accuarately because it should be made after partition process of beef carcasses and because it varies by cutting process especially trimming of fat (Abraham et al., 1980; Murphey et al., 1983; Schackleford et al., 1995). Therefore, effective prediction should be made at the time of carcass evaluation that has to make carcasses to have merchandizable value. A few research works reported using carcass measures on loin eye area and cold carcass weight to predict retail cut yields, but only dealt with Hanwoo carcasses (Lee et al., 2008; Park et al., 2002). According to the reports (Griffin et al., 1992; Schackleford et al., 1995) studied with various breeds differing in frame sizes or with different sex classes, nationwide prediction of retail yield percentage should cover the effects of those factors.

The objectives of this study were to investigate the relationship between cuttable parts and to derive prediction 
equation(s) for saleable retail cuts with higher accuracy than the index used currently as a national standard.

\section{Materials and Methods}

\section{Data}

First data set (data set I) to see the relationships among measurements had a total of 1,141 records of Hanwoo (Korean Native cattle) beef cut yield by parts collected from an abattoir of National Institute of Animal Science, RDA, Korea from 1996 to 2008. Another data set (data set II) to develop retail cut yield prediction models had a total of 14,389 records of beef cut yield by parts collected from 30 abattoirs and butcheries in Korea for two years from September, 2008 to April, 2009. Breed and sex of the data were Hanwoo cows and heifers (940 heads, $6.5 \%$ ), Hanwoo bulls (1,383 heads, $9.6 \%$ ), Hanwoo steers $(12,066$ heads, $65.3 \%)$ and steers of mixed breeds other than Hanwoo (14,389 heads, 18.6\%). The records consist of body weight $(\mathrm{BW}, \mathrm{kg})$ upon unloading from shipping trucks, cold carcass weight (CWT, kg), retail cut yield (Retail cut, $\mathrm{kg}$ ) and percentage (Retail cut of carcass weight, \%), total body fat (Body fat, $\mathrm{kg}$ ), bones (Bone, $\mathrm{kg}$ ), back fat thickness $(\mathrm{BF}, \mathrm{mm})$ and longissimus muscle area $\left(\right.$ LEA, $\mathrm{cm}^{2}$ ). The latter two measures (BF, LEA) were estimated by a beef carcass grading specialist from Korea Institute for Animal Products Quality Evaluation (KAPQE), Korea. Beef carcass grading system in Korea has been put to practice since 1993. The system determines beef carcass grades in two categories. One is yield grade and the other is quality grade. Quality grade is assigned by combination of visual scoring of intramuscular fat deposition, muscle color, fat color, texture, and maturity. Yield grade predicts the amount of saleable beef cuts and the grade of which is assigned by the index combining measures of cold carcass weight, loin eye area and back fat thickness. KAPQE grading specialists determine the final carcass quality and yield grades by sight and measurement on the cut surface between the last rib and the first lumbar vertebrae following the standard carcass grading rules of Korea. Cold carcass weights were measured after slaughter and chilled over night in a refrigerator. Deboning and partition of carcass were made by the technicians of the same abattoir one day after slaughter.

\section{Statistical analysis}

Summary statistics and Pearson's simple correlation coefficients between carcass and cut measures were estimated by UNIVARIATE and COR procedures of SAS
(2004). Parameters of generalized regression coefficients were estimated by GLM procedures of SAS (2004) fitting models with fixed class effects of breed, sex, and an interaction term between abattoirs and butcheries and three covariate terms (CWT, LEA, BF). Models were compared by their component variables with whole data set. Also separate models by sex classes and by breed groups were compared. From univariate study, extreme right skewness was observed especially for subcutaneous back fat thickness of cow and bull carcasses. Therefore, models for back fat thickness before and after log transformation were compared. Prediction error was calculated as deviation of predicted value from measured values and its mean and variances (prediction error variance, PEV) were estimated with MEANS procedure. Arithmetic means were separated by t-tests and Duncan's multiple range tests at $95 \%$ confidence levels.

\section{Results and Discussion}

\section{Relationships among Measurements}

All the variables except back fat thickness distributed quite normally. However, back fat thickness was right skewed, which support the evidence of cattle sellers' feeding style to have thinner back fat to get higher yield grades. This was more evident in cases of female carcasses, which also is suggestive of lack of fattening procedure of females, mostly old cows.

Table 1 shows means and standard deviatons of the variables by sexes (data set I). Bull carcasses were the heaviest of all sexes and their retail cut percentage was also the highest as was the case of Lee et al. (2008) or Park et al. (2002). Female carcass yielded higher retail cut percentage than steer carcass even with lighter body or carcass weight. This must be due to thinner back fat thickness of female carcass than of steer carcass.

Table 2 and 3 show the simple correlation structure between measures by sexes. Regardless of the sexes of carcasses (Table 2), retail cut percentage was highly correlated with body fat reserves and back fat thickness. However, the amount of retail cut was highly correlated with fasted body weight (BW) or with cold carcass weight (CWT). The same trend in correlation was also found in separate analyses by sexes. Higher relationship between the amount of body fat and retail cut percentage than that between back fat thickness and retail cut percentage was consistantly observed in all sexes, which is well coincide with the result of Diklean et al. (1998) with breeds other than Hanwoo. However, the amount of body fat is not 
Table 1. Means and standard deviations of measures ${ }^{1)}$ by sexes of Hanwoo

\begin{tabular}{lllcccll}
\hline \hline & $\mathrm{N}$ & CWT $(\mathrm{kg})$ & Retail cut $(\mathrm{kg})$ & Retail cut $(\%)$ & Body fat $(\mathrm{kg})$ & BF $(\mathrm{mm})$ & LEA $\left(\mathrm{cm}^{2}\right)$ \\
\hline All sexes & 1141 & $353.4 \pm 66.6$ & $240.5 \pm 44.5$ & $68.2 \pm 4.2$ & $71.1 \pm 26.8$ & $8.4 \pm 4.5$ & $84.3 \pm 12.9$ \\
Female & 196 & $275.2 \pm 46.2^{\mathrm{B}}$ & $189.6 \pm 30.6^{\mathrm{C}}$ & $69.1 \pm 3.9^{\mathrm{B}}$ & $50.1 \pm 20.7^{\mathrm{C}}$ & $8.1 \pm 3.7^{\mathrm{B}}$ & $73.0^{\mathrm{C}} \pm 10.9^{\mathrm{C}}$ \\
Bulls & 378 & $374.4 \pm 58.6^{\mathrm{A}}$ & $264.8 \pm 38.9^{\mathrm{A}}$ & $71.0 \pm 4.1^{\mathrm{A}}$ & $63.6 \pm 25.2^{\mathrm{A}}$ & $6.7 \pm 4.3^{\mathrm{C}}$ & $89.4 \pm 12.6^{\mathrm{A}}$ \\
Steers & 567 & $366.5 \pm 57.7^{\mathrm{A}}$ & $241.8 \pm 36.9^{\mathrm{B}}$ & $66.1 \pm 3.2^{\mathrm{C}}$ & $83.4 \pm 23.0^{\mathrm{B}}$ & $9.6 \pm 4.5^{\mathrm{A}}$ & $84.8^{\mathrm{A}} \pm 11.3^{\mathrm{B}}$ \\
\hline
\end{tabular}

${ }^{15} \mathrm{BW}$, body weight before slaughter; CWT, cold carcass weight; BF, back fat thickness; LEA, loin eye area.

${ }^{\mathrm{ABC}}$ Means with different superscripts differ significantly $(p<0.05)$ within the same column (means with ${ }^{\mathrm{A}}$ the largest and those with ${ }^{\mathrm{C}}$ the smallest in order).

Table 2. Correlation coefficients (Pearson's) between measures ${ }^{1)}$ for all sexes (above diagonals) and for bulls (below diagonals)

\begin{tabular}{|c|c|c|c|c|c|c|c|c|}
\hline "Bull/overall & BW (kg) & "CWT (kg) & Retail cut (kg) & Retail cut (\%) & Body fat (kg) & "Bone (kg) & BF (mm) & LEA $\left(\mathrm{cm}^{2}\right)$ \\
\hline BW & 1 & 0.97 & 0.95 & -0.13 & 0.63 & 0.79 & 0.32 & 0.69 \\
\hline CWT & 0.96 & 1 & 0.94 & -0.24 & 0.72 & 0.72 & 0.36 & 0.71 \\
\hline Retail cut & 0.93 & 0.93 & 1 & 0.09 & 0.46 & 0.85 & 0.15 & 0.75 \\
\hline Retail cut \% & -0.27 & -0.37 & 0.00 & 1 & -0.82 & 0.33 & -0.62 & 0.05 \\
\hline Body fat & 0.58 & 0.69 & 0.38 & -0.91 & 1 & 0.12 & 0.66 & 0.38 \\
\hline Bone & 0.76 & 0.68 & 0.83 & 0.26 & 0.02 & 1 & -0.04 & 0.57 \\
\hline $\mathrm{BF}$ & 0.38 & 0.43 & 0.19 & -0.68 & 0.72 & -0.02 & 1 & 0.17 \\
\hline LEA & 0.58 & 0.61 & 0.64 & -0.04 & 0.31 & 0.43 & & 1 \\
\hline
\end{tabular}

${ }^{10} \mathrm{BW}$, body weight before slaughter; CWT, cold carcass weight; BF, back fat thickness; LEA, loin eye area.

Table 3. Correlation coefficients (Pearson's) between measures ${ }^{1)}$ for females (above diagonals) and for steers (below diagonals)

\begin{tabular}{lcccccccc}
\hline \hline Steer/Female & BW $(\mathrm{kg})$ & CWT $(\mathrm{kg})$ & Retail cut $(\mathrm{kg})$ Retail cut $(\%)$ & Body fat $(\mathrm{kg})$ & Bone $(\mathrm{kg})$ & BF $(\mathrm{mm})$ & LEA $\left(\mathrm{cm}^{2}\right)$ \\
\hline BW & 1 & 0.94 & 0.96 & -0.08 & 0.51 & 0.61 & 0.44 & 0.64 \\
CWT & 0.97 & 1 & 0.94 & -0.28 & 0.71 & 0.42 & 0.51 & 0.69 \\
Retail cut & 0.96 & 0.95 & 1 & 0.05 & 0.44 & 0.62 & 0.37 & 0.73 \\
Retail cut \% & -0.12 & -0.24 & 0.07 & 1 & -0.85 & 0.54 & -0.48 & 0.11 \\
Body fat & 0.70 & 0.80 & 0.57 & -0.76 & 1 & -0.28 & 0.59 & 0.30 \\
Bone & 0.84 & 0.78 & 0.87 & 0.22 & 0.32 & 1 & 0.01 & 0.40 \\
BF & 0.38 & 0.44 & 0.28 & -0.55 & 0.65 & 0.09 & 1 & 0.27 \\
LEA & 0.65 & 0.67 & 0.70 & 0.01 & 0.44 & 0.54 & 0.23 & 1 \\
\hline
\end{tabular}

${ }^{11} \mathrm{BW}$, body weight before slaughter; CWT, cold carcass weight; BF, back fat thickness; LEA, loin eye area.

weighed at the time of carcass grading or while on slaughter process. The second important variable that is correlated with percent retail cut was carcass weight (CWT) which is measurable after slaughter before grades are assigned for each carcass. The other variable that is correlated with percent retail cut and is measurable at the time of grading is loin eye area (LEA). But the relationship was very small for all sexes. This was consistent with the result of Jones et al. (1990) or Lee et al. (2010). But the result was far lower than that of Harris et al. (1995) with Japanese yield grading system (0.78). Currently in Korea, carcass yield grades are assigned by an index composed of those three measurable variables-CWT, BF and LEA. Therefore, further researches on accuracy of prediction of percent retail cuts should follow because the relationships of the yield values with those three measurable traits were variable by sexes of the carcasses as pointed by Jones et al. (1990) and Reverter et al. (1999).

\section{Prediction models}

Table 4 shows summary statistics of carcass parameters by sex classes and by breed groups (data set II). Generally other breeds (mostly Holstein) grow faster than Hanwoo cattle and can be slaughtered in younger age because most Holstein steers are slaughtered at around 22 months of age while Hanwoo steers are killed at around 30 months of age. Therefore, carcass weight and retail cut yields of other breeds are greater with thinner back fat thickness than those of Hanwoo cattle. But size of loin eye muscle is much smaller which support the evidence of much younger age at slaughter. Lee et al. (2008), Jones et al. (1990), and Park et al. (2002) reported that sex of Hanwoo cattle is a significant source of variation determining retail cut percentages. And Diklean et al. (1998)'s report also revealed significance of sex effect in other breeds as well. Reverter et al. (1999) had observed the retail cut percentage by breeds of different origins. Effect 
Table 4. Means and standard deviation of the carcass and beef cut measures ${ }^{1)}$ by sex and by breed

\begin{tabular}{|c|c|c|c|c|c|c|c|}
\hline Breed & sex & $\mathrm{N}$ & CWT (kg) & Retail cut (kg) & Retail cut (\%) & $\mathrm{BF}(\mathrm{mm})$ & LEA $\left(\mathrm{cm}^{2}\right)$ \\
\hline \multirow{3}{*}{ Hanwoo } & Steers & 9391 & $405.6 \pm 39.8^{\mathrm{B}}$ & $254.1 \pm 26.3^{\mathrm{B}}$ & $62.7 \pm 3.0^{\mathrm{C}}$ & $12.4 \pm 4.5^{\mathrm{A}}$ & $88.4 \pm 9.3^{\mathrm{A}}$ \\
\hline & Female & 940 & $335.5 \pm 35.2^{\mathrm{D}}$ & $208.1 \pm 22.9^{\mathrm{D}}$ & $62.1 \pm 2.8^{\mathrm{D}}$ & $10.4 \pm 4.0^{\mathrm{B}}$ & $76.4 \pm 8.9^{\mathrm{C}}$ \\
\hline & Bulls & 1383 & $376.2 \pm 44.0^{\mathrm{C}}$ & $251.6 \pm 31.1^{\mathrm{C}}$ & $66.9 \pm 3.7^{\mathrm{A}}$ & $5.6 \pm 2.8^{\mathrm{D}}$ & $86.2 \pm 9.8^{\mathrm{B}}$ \\
\hline Other & Steers & 2675 & $410.2 \pm 35.5^{\mathrm{A}}$ & $259.9 \pm 23.0^{\mathrm{A}}$ & $63.4 \pm 3.0^{\mathrm{B}}$ & $7.0 \pm 3.0^{\mathrm{C}}$ & $74.2 \pm 7.7^{\mathrm{D}}$ \\
\hline \multicolumn{8}{|c|}{ p-values from contrasts } \\
\hline \multicolumn{2}{|c|}{ Hanwoo vs. other } & \multicolumn{2}{|r|}{$<0.0001$} & $<0.0001$ & $<0.0001$ & $<0.0001$ & $<0.0001$ \\
\hline \multicolumn{2}{|c|}{ Hanwoo: female vs. bulls } & \multicolumn{2}{|r|}{$<0.0001$} & $<0.0001$ & $<0.0001$ & $<0.0001$ & $<0.0001$ \\
\hline \multicolumn{2}{|c|}{ Hanwoo: female vs. steers } & \multicolumn{2}{|r|}{$<0.0001$} & $<0.0001$ & 0.0072 & 0.6741 & $<0.0001$ \\
\hline \multicolumn{2}{|c|}{ Hanwoo: bulls vs. steers } & \multicolumn{2}{|r|}{$<0.0001$} & $<0.0001$ & $<0.0001$ & $<0.0001$ & $<0.0001$ \\
\hline \multicolumn{2}{|c|}{ Steer: Hanwoo vs. other } & \multicolumn{2}{|r|}{$<0.0001$} & 0.5933 & $<0.0001$ & $<0.0001$ & $<0.0001$ \\
\hline
\end{tabular}

${ }^{11} \mathrm{BW}$, body weight before slaughter; CWT, cold carcass weight; BF, back fat thickness; LEA: loin eye area.

${ }^{A B C D}$ Means with different superscripts differ significantly $(p<0.05)$ within the same column (means with ${ }^{\mathrm{A}}$ the largest and those with ${ }^{\mathrm{C}}$ the smallest in order).

of sex can be understood as differences in feeding program. Breed difference can be understood as differences in market ages, especially when Hanwoo and Holstein breeds are combined into consideration together. As pointed by Henderson et al. (1966), different population, breed group in this study, can have different relationship between carcass measures. Opposed to the observation by Griffin et al. (1992), Holstein steers (other breed group) yielded similar and somewhat higher retail cut percentage than Hanwoo steers. This might be due to longer feeding of Hanwoo steers and therefore covering thicker back fat at finishing.

Shackleford et al. (1995), in their study with Germplasm Utilization Project data, compared variaous parameters concluded that rib dissection traits as used in Korean yield grading system were the best predictors.

Tables 5 and 6 show the regression coefficients of the three covariates to predict retail cut percentages by breeds and sexes. Error mean square was chosen as a criteria to selection of the best model. Table 5 fits models with back fat thickness as a direct linear variable while Table 6 fits models with back fat thickness as a logarithmic transformed variable to make it normalized. Among the sex classes, steer class of Hanwoo best fit the model while steers of other breed group did not fit well. This might be due to wide range of breed composition and feeding system. Among the predictor variables, back fat thickness was the most important while carcass weight or longissimus dorsi area contributed very small variation as understood by partial regression coefficients as was in the literature (Diklean et al., 1998; Griffin et al., 1992; Lee et al., 2008; Henderson et al., 1966) .

Model fitness as determined by either $\mathrm{R}^{2}$ or $\sqrt{M S E}$ became a little better by taking log value of BF but not quite considerably. However, prediction error variances of our new models were much smaller than the model

Table 5. Estimates for prediction ${ }^{1)}$ of \% retail cut by breeds and sexes

\begin{tabular}{cccccccccc}
\hline \hline breed & sex & $\mathrm{N}$ & $\mathrm{R}^{2}$ & $\sqrt{M S E}^{3)}$ & Intercept & CWT & BF & LEA \\
\hline 1 & 1 & 925 & 0.429 & 2.087 & 62.9785 & -0.0112 & -0.1871 & 0.0979 \\
1 & 2 & 1344 & 0.535 & 2.541 & 70.1382 & -0.0091 & -0.4196 & 0.0652 \\
1 & 3 & 9259 & 0.540 & 2.034 & 68.0800 & -0.0179 & -0.1902 & 0.0933 \\
2 & 3 & 2647 & 0.154 & 2.759 & 68.1082 & -0.0216 & -0.2084 & 0.0679 \\
\hline
\end{tabular}

${ }^{1}$ Prediction Model: [\%retail cut], $\beta_{0}+\left[\right.$ house*butcherer] $\left.+\beta_{1}[\mathrm{CWT}]+\beta_{2}[\mathrm{BF})\right]+\beta_{3}[\mathrm{LEA}]+\varepsilon$.

${ }^{2}$ Breed: 1, Hanwoo; 2, other; sex: 1, female; 2, bull; 3, steer.

$\sqrt{M S E}$, square root of MSE (error mean square).

Table 6. Estimates for prediction ${ }^{1)}$ of $\%$ retail cut by breeds and $\operatorname{sexes}^{2)}$ using $\log$ transformed value of back fat thickness

\begin{tabular}{ccccccccc}
\hline \hline breed & sex & $\mathrm{N}$ & $\mathrm{R}^{2}$ & $\sqrt{M S E}$ & Intercept & $\mathrm{CWT}$ & $\log (\mathrm{BF})$ & $\mathrm{LEA}$ \\
\hline 1 & 1 & 925 & 0.436 & 2.075 & 65.3304 & -0.0108 & -1.9752 & 0.0974 \\
1 & 2 & 1344 & 0.542 & 2.522 & 71.9158 & -0.0085 & -2.6833 & 0.0659 \\
1 & 3 & 9259 & 0.538 & 2.040 & 71.1873 & -0.0180 & -2.1900 & 0.0939 \\
2 & 3 & 2647 & 0.157 & 2.755 & 69.5805 & -0.0212 & -1.9378 & 0.0972 \\
\hline
\end{tabular}


Table 7. Prediction error variances (PEV) from indices ${ }^{1)}$ for all breeds and sexes ${ }^{2}$

\begin{tabular}{ccc}
\hline \hline Variable & $\mathrm{N}$ & PEV \\
\hline penow & 14389 & 11.75251 \\
pe1_3 & 14389 & 8.81203 \\
pe2_3 & 14389 & 8.544518 \\
pe1_4 & 14389 & 8.767299 \\
pe2_4 & 14389 & 8.535938 \\
\hline
\end{tabular}

${ }^{1)}$ inow $=\mathbf{6 8 . 1 8 4}-\mathbf{0 . 0 2 4} *$ cwt $-\mathbf{0 . 6 2 5} *$ bf $+\mathbf{0 . 1 3 0} *$ LEA (current yield index) and penow $=p$ retailcut-inow, i1 $3=\mathbf{6 8 . 2 8 0 2}-\mathbf{0 . 0 1 7 4} *$ cwt $\mathbf{- 0 . 1 9 1 6} *$ bf $+\mathbf{0 . 0 8 8 2} *$ LEA and pe1_3 = p_retailcut-i1_3, i2 $3=$ 70.9531 - 0.0168* ${ }^{*}$ wt $-2.1101 * \operatorname{logbf}+\mathbf{0 . 0 8 8 7} *$ LEA and pe $2 \_3=$ p_retailcut-i2 3, i1 $4=\mathbf{6 8 . 3 3 6 8}-\mathbf{0 . 0 1 7 1} *$ cwt- $0.2036 *$ bf $+0.0879 *$ LEA and pe1_4=p_retailcut-i1_4, i2_4 $=\mathbf{7 0 . 9 9 1 7}-\mathbf{0 . 0 1 6 8} *$ cwt 2.1338 $* \operatorname{logbf}+\mathbf{0 . 0 8 8 6} *$ LEA and pe $2 \_4=$ p_retailcut-i2_4.

${ }^{2}$ Breed: 1, Hanwoo; 2, other; sex: 1, female; 2 , bull; 3, steer.

used as a current national standard (Table 7). Indices i1_3 and i2_3 are the index models fit by a subset of data used in this study $(\mathrm{N}=12,247)$ without or with log transformation of BF, respectively. And indices i1_4 and i2_4 are the index models fit by full set of data $(\mathrm{N}=14,389)$ without or with log transformation, respectively. All of these four models predict retail cut percentage regardless of the effect of breed and sex.

To conclude, average statistics of carcass and beef cut measures and the relationship between variables were investigated. The investigation was made with Hanwoo carcasses of three different sexual originations. Percentage of carcass that is saleable as edible meat, percent retail cut, was highly correlated with the amount of body fat and back fat thickness while the amount of saleable cut is highly correlated with live body weight before slaughter or with cold carcass weight. We suggest accounting for sexes of the carcass when to predict retail cut percentage with higher accuracy.

Covariance models were developed to increase predictability of beef carcass yield index. Breed specific indices were compared with or without normalization of back fat thickness. And prediction error variances were compared between currently used model and newly developed models independent of breed and sex. We suggest to use new prediction models, preferably the model with log transformation of back fat thickness because this might be more powerful statistically to accommodate future changes in back fat control.

\section{Acknowledgement}

Authors thank data collection efforts of KAPQE, Korea and participant members of abattoirs and butcheries.

\section{References}

1. Abraham, H. C., Murphey, C. E., Cross, H. R., and Smith, G. C. (1980) Factors affecting beef carcass cutability: An evaluation of the USDA yield grades for beef. J. Anim. Sci. 50, 841-851.

2. Diklean, M. E., Cundiff, L. V., Gregory, K. E., Kemp, K. E., and Koch, R. M. (1998) Relative contribution of subcutaneous and intermuscular fat to yields and predictability of retail product, fat trim, and bone in beef carcasses. J. Anim. Sci. 76, 1604-1612.

3. Griffin, D. B., Savell, J. W., Morgan, J. B., Garrett, B. P., and Cross, H. R. (1992) Estimates of subprimal yields from beef carcasses as affected by USDA grades, subcutaneous fat trim level, and carcass sex class and type. J. Anim. Sci. 70, 24112430.

4. Harris, J. J., Lunt, D. K., Savell, J. W., Hawkins, E. W., and Orme, L. E. (1995) Relationjship between USDA and Japanese beef grades. Meat Sci. 39, 87-95.

5. Henderson, D. W., Goll, D. E., and Kline, E. A. (1966) Relationships of muscling and finish measurements from three different groups of beef carcasses with carcass yield. J. Anim. Sci. 25, 323-328.

6. Jones, D. K., Savell, J. W., and Cross, H. R. (1990) The influence of sex-class, USDA yield grade and USDA quality grade on seam fat trim from the primal of beef carcasses. $J$. Anim. Sci. 68, 1987-1991.

7. Lee, J. M., Hah, K. H., Kim, J. H., Cho, S. H., Seong, P. N., Jung, M. O., Cho, Y. M., Park, B. Y., Kim, D. H., and Ahn, J. N. (2008) Study on the carcass yield grade traits and predictin of retail product weight in Hanwoo beef. Korean $J$. Food Sci. Ai. Resour. 28, 604-609.

8. Lee, J. M., Choe, J. H., Lee, H. K., Na, J. C., Kim, Y. H., Cheon, D. W., Sea, S. C., and Hwang, K. S. (2010) Effect of quality grades on carcass characteristics, physico-chemical and sensory traits of longissimus dorsi in Hanwoo. Korean $J$. Food Sci. Ai. Resour. 30, 495-503.

9. Murphey, C. E., Recio, H. A., Stiffler, D. M., Smith, G. C., Savell, J. W., and Wise, J. W. (1983) Effects of trimming fat from beef carcasses on the accuracy of determining USDA yield grade. J. Anim. Sci. 57, 349-354.

10. Park, G. B., Moon, S. S., Ko, Y. D., Ha, J. K., Lee, J. K., Chang, H. H., and Joo, S. T. (2002) Influence of sluaghter weight and sex on yield and quality grades of Hanwoo (Korean native cattle) carcasses. J. Anim. Sci. 80, 129-136.

11. Reverter, A., Johnston, D. J., Stephens, E., and Perry, D. (1999) Development of a prediction equation for retail beef yield precent to be used in national genetic evaluation schemes. Proceed Assoc. Advmnt. Anim. Bree. Genet. 13, pp. 381-384.

12. SAS/STAT ${ }^{\circledR} 9.1$ User's Guide (2004) SAS Institute Inc. Cary, NC, USA, ISBN 1-59047-243-8.

13. Schackelford, S. D., Cundiff, L. V., Gregory, K. E., and Koohmaraie, M. (1995) Predicting beef carcass cutability. $J$. Anim. Sci. 73, 406-413.

(Received 2010.9.8/Revised 2010.10.15/Accepted 2010.10.19) 